\title{
A further insight into the metabolic profile of the nuclear receptor Rev-erb agonist, SR9009
}

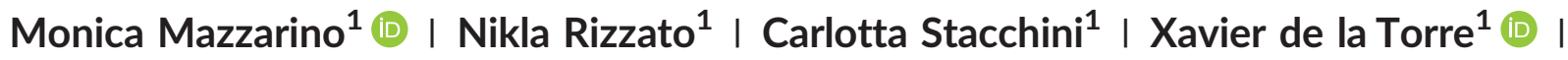 \\ Francesco Botrè ${ }^{1,2}$
}

${ }^{1}$ Laboratorio Antidoping, Federazione Medico Sportiva Italiana, Rome, Italy

${ }^{2}$ Dipartimento di Medicina Sperimentale, "Sapienza" Università di Roma, Rome, Italy Correspondence

Monica Mazzarino, Laboratorio Antidoping, Federazione Medico Sportiva Italiana, Largo Giulio Onesti, 1, 00197 Rome, Italy. Email: monica.mazzarino@gmail.com

Funding information Italian Department of Health

\begin{abstract}
The reactions involved in the metabolic pathways of SR9009 were characterized by liquid chromatography-mass spectrometry (LC-MS) to identify the most appropriate marker(s) of use. The effects of gender, genetic polymorphism, and drug-drug interaction on the metabolic profile of SR9009 were also evaluated. In vitro approaches were based on the use of human liver microsomes and cytochrome P450 isoforms. Sample preparation included an enzymatic hydrolysis (performed only for the phase II investigation) followed by liquid-liquid extraction. The chromatographic separation was carried out using a reverse-phase column; detection was performed by either a triple-quadrupole or a time-of-flight system in positive electrospray ionization and different acquisition modes. In the presence of human liver microsomes, SR9009 was biotransformed to 13 metabolites by CYP3A4, CYP3A5, CYP2C19, and CYP2D6 isoenzymes. The reactions included hydroxylation, de-alkylation, oxidation, and combinations thereof, the de-alkylated metabolites being the most abundant. Once formed the mentioned metabolites underwent glucuronidation. Concerning the effects of gender, genetic polymorphism, and drug-drug interaction on the metabolic profile of SR9009, our observation have shown the following: (a) No significant alterations were measured between female and male, (b) significant differences were registered using either the CYP2D6 or CYP2C19 allelic variants, and finally (c) significant alterations were registered in the presence of ketoconazole, miconazole, fluoxetine, nefazodone and paroxetine; moderate variation were instead registered with fluconazole, itraconazole, gestodene, and levonorgestrel. This observation put in evidence the importance to take into account both genetic polymorphism and drug-drug interaction to select the most appropriate marker(s) of use in doping analysis.
\end{abstract}

\section{KEYWORDS}

anti-doping analysis, antifungals, drug-drug interaction, genetic polymorphism, Rev-erb SR9009

\section{I INTRODUCTION}

The recent discovery that Rev-erbs are ligand-regulated receptors whose repressive activity is regulated by the endogenous porphyrinligand, heme, has stimulated the development of synthetic ligands, ${ }^{1-5}$ opening up the possibility of targeting them for therapeutic purposes. $^{6-9}$ Several novel compounds were designed and synthesized to interact with the Rev-erb receptor, the first being GSK4112 (also known as SR6452). ${ }^{7-9}$ This synthetic ligand was demonstrated to act as an agonist of the Rev-erb receptors, mimicking the action of the endogenous ligand, in cell-based assays, but did not exhibit a favorable pharmacokinetic profile. In an attempt to improve potency, efficacy, and pharmacokinetics properties, two Rev-erb agonists (denoted as SR9009 and SR9011) were proposed ${ }^{7-9}$ Both in vitro and in vivo 
animal studies reported the ability of SR9009 and SR9011 (a) to increase basal oxygen consumption, mitochondrial content, glucose, and fatty acid oxidation in the skeletal muscle; and (b) to decrease lipogenesis, cholesterol, and bile acid synthesis in the liver and lipid storage in the white adipose tissue. ${ }^{7-12}$ Although both SR9009 and SR9011 are still undergoing clinical evaluation and are therefore not approved for therapeutic use, their distribution through black market channels, mainly within the bodybuilding community, has been reported. ${ }^{13}$ In view of this, the World Anti-Doping Agency (WADA) has included the Rev-erb agonists on the list of doping substances and methods. Originally banned as non-approved substances (Class SO of the WADA Prohibited List International Standard), they have been moved to class S4 "Hormone and Metabolic Modulators" since January $1,2018 .^{14}$

Previous investigators have carried out in vitro metabolism studies, identifying eight metabolites ${ }^{15,16}$ The metabolic conversion resulted in the loss of the (5-nitrothiophen-2-yl) methyl group, of the (4-chlrophenyl) methyl group, or of the ([(1-ethoxycarbonyl) pyrrolidin-3-yl] methyl portion followed by hydroxylation or oxidation (Figure 1 shows the chemical structure of SR9009). In vivo studies by Sobolevsky et al are partially confirmed by the results obtained in vitro indicating the loss of the (4-chlrophenyl) methyl group and of the ([(1-ethoxycarbonyl)pyrrolidin-3-yl] methyl portion (Figure 1) as the most important biotransformation pathways of SR9009 in human. ${ }^{15}$

The goal of the present work is to extend the knowledge about the metabolic profile of SR9009, also considering the effects of gender, genetic polymorphism, and drug-drug interaction. For this purpose, following similar studies performed on other classes of substances prohibited in sport, ${ }^{17-19}$ in vitro metabolism studies were performed to characterize (a) the phase I and phase II metabolic pathways; (b) the isoenzymes involved in the phase I biotransformation reactions; and (c) the potential alteration of the above caused by gender differences, genetic polymorphism, and drug-drug interaction. The non-prohibited drugs were selected from those most commonly used by the athletes (according to the information available on the doping control form) and/or reported to be inhibitors of the CYP450 system $^{20-23}$ Specifically, four antifungals (ketoconazole, miconazole, itraconazole, and fluconazole), three antidepressants (paroxetine, fluoxetine, and nefazodone), three non-steroidal anti-inflammatory drugs (NSAIDs; ibuprofen, ketoprofen, and diclofenac), two H2 receptor antagonists (ranitidine and cimetidine), and two steroidal progestins (gestodene and levonorgestrel) were considered. ${ }^{20-23}$

Given the difficulty in obtaining permission to perform excretion studies on humans (especially when involving the co-administration of two or more active principles), our study was based on in vitro experiments carried out using human liver microsomes (HLM) and different recombinant isoforms of Cyp450. The ability of the in vitro models and protocols described in this study to reproduce the human metabolic pathways was then confirmed by analyzing a urine sample collected after oral administration of a nutritional supplement containing SR9009, kindly supplied by the World Association of Anti-Doping Scientists (WAADS).

\section{2 | EXPERIMENTAL}

\section{1 | Chemicals and reagents}

SR9009 was supplied by Tocris Bioscience (Bristol, UK). 17amethyltestosterone (used as internal standard), ketoconazole, miconazole, itraconazole, fluconazole, ibuprofen, diclofenac, ketoprofen, fluoxetine, paroxetine, nefazodone, ranitidine, cimetidine, gestodene, and levonorgestrel (used as inhibitors) were supplied by Sigma-Aldrich (Milan, Italy). Certified standards of metabolites M2 and M9 were from NARL (NMIA, National Measurement Institute Australia, Sydney, Australia). Formic acid, sodium phosphate, sodium hydrogen phosphate, acetonitrile, methanol, dimethylsulfoxide (DMSO), and tert-butyl methyl-ether were all of analytical grade and provided by SigmaAldrich (Milan, Italy). The ultrapurified water used was of Milli-Q-grade (Millipore Italia, Vimodrone, Milan, Italy).

The enzyme $\beta$-glucuronidase (from $E$. coli) used for the enzymatic hydrolysis of conjugates, was purchased from Roche (Monza, Italy). The enzymatic proteins (CYP recombinant isoforms CYP1A2, CYP3A4, CYP3A5, CYP2B6, CYP2C8, CYP2C9, CYP2C19, and CYP2D6, as well as HLM pooled from 20 Caucasian mixed male and female donors of different ages, HLM from female donors, and HLM from male donors) and all the reagents for the in vitro assays (sodium phosphate and tris$\mathrm{HCl}$ buffers, magnesium chloride hexahydrate, uridine $5^{\prime}$-diphospho- $a$-D-glucuronic acid, alamethicin, glucose-6-phosphate, glucose-6-phosphate dehydrogenase, nicotinamide adenine dinucleotide phosphate, and CYP450 oxidoreductase) were supplied by BD

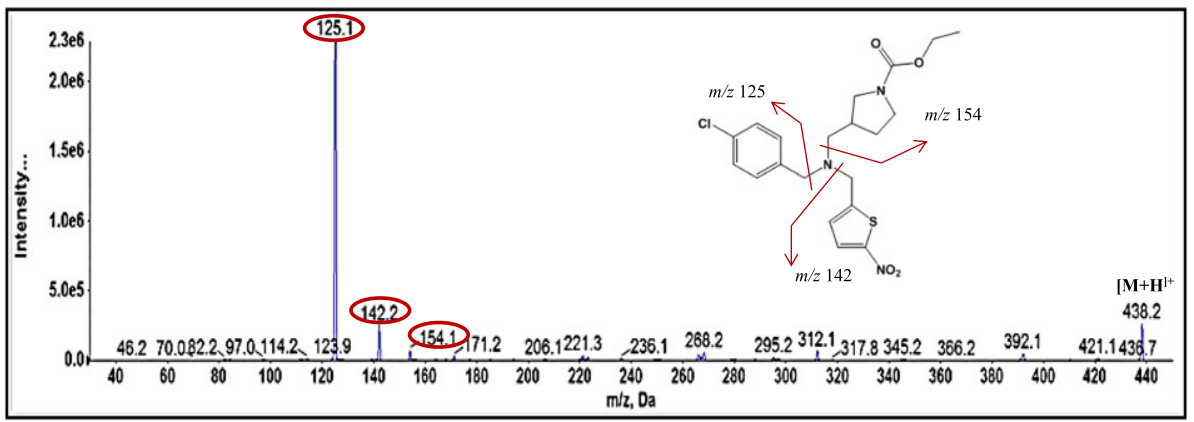

FIGURE 1 Product ion spectra obtained at collision energy $30 \mathrm{eV}$, molecular structure, fragmentation pathways and structural markers of SR9009 
Biosciences (Milan, Italy). CYP2C19 and CYP2D6 allelic variants were supplied from XenoTech (Kansas City, MO, USA). The excretion urine, collected after oral administration of a nutritional supplement containing SR9009, was kindly supplied by WAADS.

\section{2 | In vitro protocols}

\subsection{1 | Phase I metabolism}

All incubation conditions were optimized (protein and substrate concentrations, buffer and solvent types, incubation time) considering the protocols already used in previous studies carried out in our laboratory. ${ }^{17-19,24}$ Different solvents (methanol, DMSO, and acetonitrile), buffers (phosphate and tris- $\mathrm{HCl}$ ), $\mathrm{pH}$ values $(5.0,7.4,8.0)$, concentration of SR9009 $(0.1,0.5$, and $1 \mathrm{mg} / \mathrm{mL})$ and of the enzymatic proteins $(0.1,0.2,0.5$, and $1.0 \mathrm{mg} / \mathrm{mL})$, and different incubation time $(30,60$, $120,240,480$, and 720 minutes) were evaluated. The final incubation medium also contained $3.3 \mathrm{mM}$ magnesium chloride, 1.3mM NADP ${ }^{+}$, 3.3mM glucose-6-phosphate, and $0.4 \mathrm{U} / \mathrm{mL}$ glucose-6-phosphate dehydrogenase in a total volume of $250 \mu \mathrm{L}$. Samples were prewarmed at $37^{\circ} \mathrm{C}$ for 5 minutes and the reactions were started with the addition of the appropriate enzymatic proteins (HLM or CYP recombinant isoforms). After incubation at $37^{\circ} \mathrm{C}, 250 \mu \mathrm{L}$ of ice-cold acetonitrile were added to stop the reaction. The samples were then transferred into an ice bath for 3 minutes for further precipitation of the proteins in the assay medium. The supernatant was subsequently separated from the precipitate by centrifugation at $21000 \mathrm{~g}$ at room temperature for 10 minutes. Each set of assays also included a negative control sample, containing all reaction mixture components except the enzymatic protein to monitor the potential non-enzymatic reactions within the incubation period. Each incubation was processed in duplicate.

\subsection{2 | Phase II metabolism}

To study the glucuronidation reactions, $8 \mathrm{mM}$ magnesium chloride and $25 \mu \mathrm{g} / \mathrm{mL}$ of alamethicin were added to the phase I reaction mixture described in the experimental section. The phase I reaction was then initiated by adding $0.5 \mathrm{mg} / \mathrm{mL}$ of $\mathrm{HLM}$ in an incubation volume of $250 \mu \mathrm{L}$. After 1 minute at $37^{\circ} \mathrm{C}, 2 \mathrm{mM}$ of the cofactor uridine $5^{\prime}$ di-phospho-a-D-glucuronic acid was added and the phase II metabolism was run for 4 hours at $37^{\circ} \mathrm{C}$. Proteins were then precipitated using the protocols described for the phase I metabolism. Negative control samples (either without proteins or without the substrate) were prepared and each incubation was processed in duplicate.

\section{3 | Sample preparation}

Sample pre-treatment was based on a previously described procedure. ${ }^{24-26}$ Briefly, both the samples obtained by the in vitro experiments (sample volume $500 \mu \mathrm{L}$ ) and the excretion urine (sample volume $1 \mathrm{~mL}$ ) were prepared as follows: $1.5 \mathrm{~mL}$ of phosphate buffer (1 $\mathrm{M}, \mathrm{pH}$ 7.4), and $50 \mu \mathrm{L}$ of the internal standard (ISTD: solution of $17 a$-methyltestosterone $10 \mu \mathrm{g} / \mathrm{mL}$ ) were added and the liquid-liquid extraction (LLE) was carried out with $7 \mathrm{~mL}$ tert-butylmethyl ether for
6 minutes on a mechanical shaker; after centrifugation, the organic layer was separated and evaporated to dryness at $40^{\circ} \mathrm{C}$; the residue was reconstituted in $50 \mu \mathrm{L}$ of mobile phase (initial composition) and an aliquot of $10 \mu \mathrm{L}$ was injected in the liquid chromatography-mass spectrometry (LC-MS) systems for the detection of phase I metabolites.

In parallel, to identify also the glucuro-conjugated metabolites, to the aqueous layer were added $50 \mu \mathrm{L}$ of $\beta$-glucuronidase from $E$. coli and $50 \mu \mathrm{L}$ of the same ISTD and the sample was then incubated for 1 hour at $55^{\circ} \mathrm{C}$. After this enzymatic hydrolysis step, the same protocol described for the detection of phase I metabolites was followed, and an aliquot of $10 \mu \mathrm{L}$ was then injected on the LC-MS systems.

\section{4 | Instrumental conditions}

\subsection{1 | LC-MS/MS conditions}

The chromatographic separation was performed using an Agilent 1200 Rapid Resolution Series HPLC pump with binary gradient system and automatic injector (Agilent Technologies S.p.A, Cernusco sul Naviglio, Milan, Italy). Reversed-phase liquid chromatography was performed using a Supelco Ascentis C18 column $(2.1 \times 100$ mm, $2.7 \mu \mathrm{m})$. The solvents used were ultrapurified water (eluent $A$ ) and acetonitrile (eluent B), both containing $0.1 \%$ formic acid. The gradient program started at $10 \% \mathrm{~B}$ and increasing to $60 \% \mathrm{~B}$ in 7 minutes, after 6 minutes, to $100 \%$ $B$ in 4 minutes. The column was flushed for 4 minutes at $100 \%$ B and finally re-equilibrated at $10 \% \mathrm{~B}$ for 4 minutes. The flow rate was set to $0.25 \mathrm{~mL} / \mathrm{min}$

Experiments were performed using a triple quadrupole instrument (API4000 ABSciex, Monza, Italy) with positive electrospray ionization (ESI). Selected reaction monitoring (SRM) experiments were performed employing collision-induced dissociation (CID) using nitrogen as collision gas at $5.8 \mathrm{mPa}$, obtained from a dedicated nitrogen generator system Parker-Balston model 75-A74, gas purity $99.5 \%$ (CPS analitica, Milan, Italy). The mass spectrometric parameters (declustering and needle voltages, gas pressure, source temperature, collision cell exit potential and collision energy) were optimized by infusion of the standard solution of SR9009 and of the metabolites $\mathrm{M} 2$ and $\mathrm{M} 9$ at a concentration of $10 \mu \mathrm{g} / \mathrm{mL}$. To this purpose, a $1 \mathrm{~mL}$ syringe operated by a syringe pump at a flow-rate of $10 \mu \mathrm{L} / \mathrm{min}$ was utilized. All aspects of instrument control, method setup parameters, sample injection and sequence operation were controlled by Analyst software version 1.6.1.

\subsection{2 | UHPLC-QTOF conditions}

The chromatographic separation was performed using an Agilent 1290 infinity II UHPLC with binary gradient system and automatic injector (Agilent Technologies S.p.A, Cernusco sul Naviglio, Milan, Italy). Reversed-phase liquid chromatography was performed using an Agilent Zorbax Eclipse plus C18 column $(2.1 \times 100$ mm, $1.8 \mu \mathrm{m})$. The solvents used were ultrapurified water (eluent A) and acetonitrile, both containing $0.1 \%$ formic acid (eluent B). The gradient program started at $5 \% \mathrm{~B}$ and increasing to $30 \% \mathrm{~B}$ in 4 minutes, to $70 \% \mathrm{~B}$ in 4 minutes, and finally to $95 \%$ B in 1 minute. The column was flushed for 3 minutes 
at $95 \% \mathrm{~B}$ and finally re-equilibrated at $5 \% \mathrm{~B}$ for 3 minutes. The flow rate was set to $0.4 \mathrm{~mL} / \mathrm{min}$.

High-resolution/high-accuracy measurements were performed on an Agilent Technologies 6545 orthogonal acceleration time-of-flight mass spectrometer, equipped with an electrospray ionization (ESI) source operating in positive ion mode. Nitrogen was used as the drying and nebulizing gas. The drying gas flow rate and temperature were
$10 \mathrm{~L} / \mathrm{min}$ and $350^{\circ} \mathrm{C}$, respectively. The nebulizer gas pressure was 45 psi. The applied capillary and the fragmentor voltages were optimized by infusion of the standard solution of SR9009 and of the metabolites M2 and M9 at a concentration of $10 \mu \mathrm{g} / \mathrm{mL}$. Mass data were generated from m/z 100 to 1100 at 9300 transients per second. All other MS parameters were automatically optimized by the daily performed instrument autotuning procedure. The mass calibration

(1)

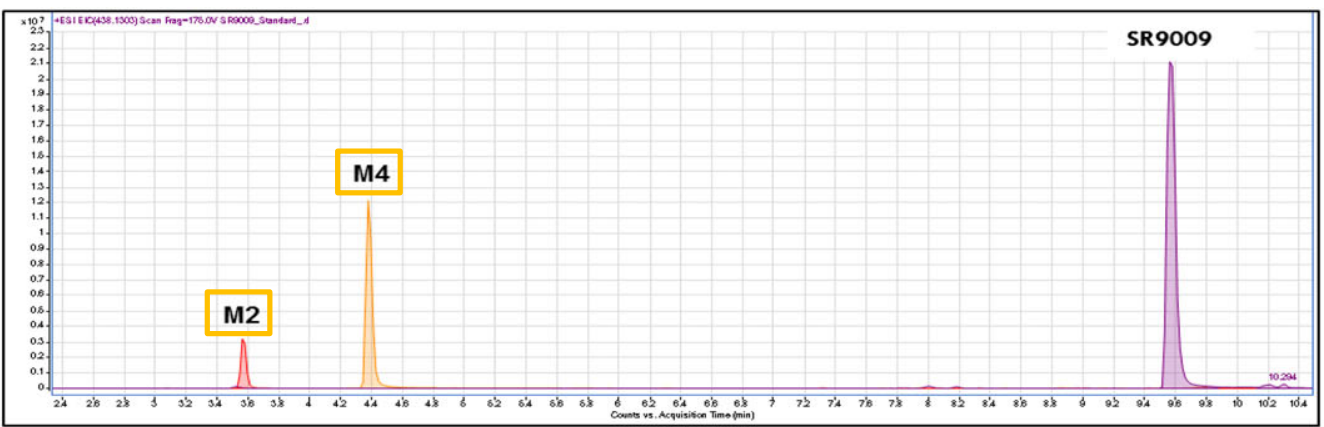

(2)

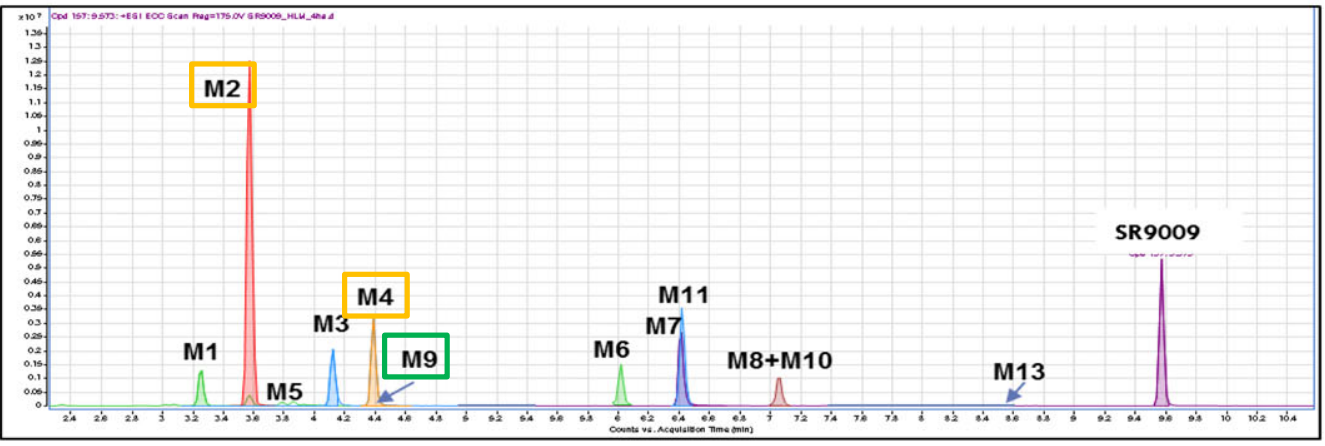

(A)

(1)

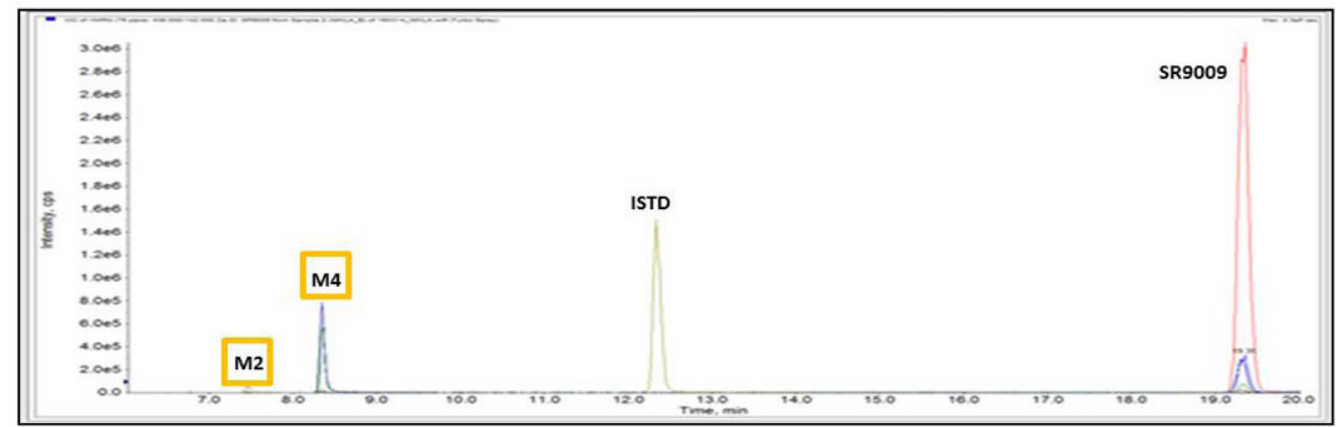

(2)

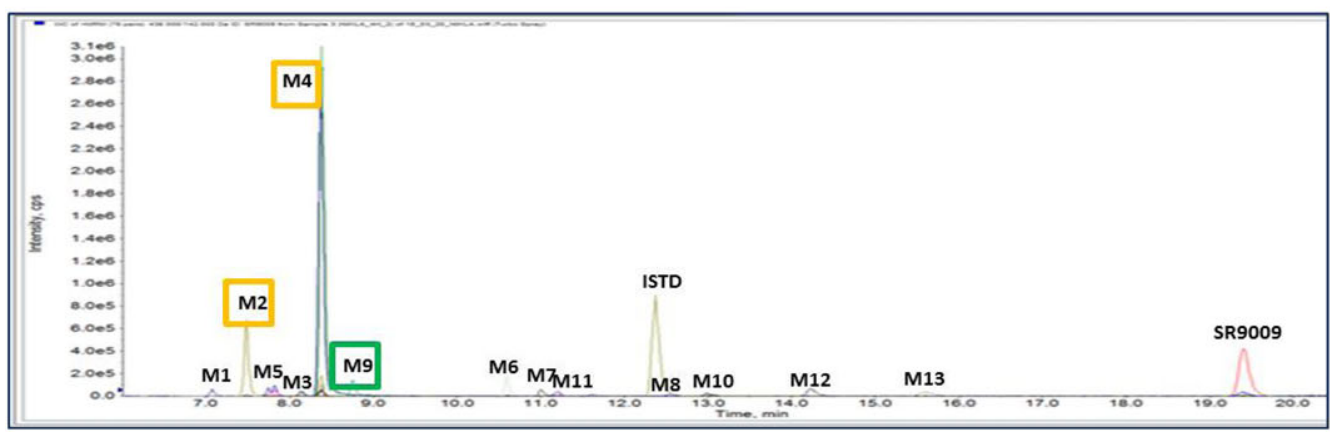

(B)

FIGURE 2 A, Extracted ion chromatograms obtained analyzing in vitro samples after incubation of SR9009 in the absence (1) and in the presence (2) of human liver microsomes, using the UHPLC-QTOF system. B, Extracted ion chromatograms obtained analyzing in vitro samples after incubation of SR9009 in the absence (1) and in the presence (2) of human liver microsomes, using the LC-MS/MS system 
was performed daily, at the beginning of every analytical session, using a calibration solution provided by the manufacturer. Purine with an $\left[\mathrm{M}+\mathrm{H}^{+}\right.$ion at $\mathrm{m} / \mathrm{z} 121.0509$ and an Agilent proprietary compound (HP0921) yielding an ion at m/z 922.0098 were simultaneously introduced via a second orthogonal sprayer, and these ions were used as internal calibrants along all the analysis. All aspects of instrument control, tuning, method setup and parameters, sample injection and sequence operation were controlled by the Agilent Technologies Mass Hunter software version B.08.00.

\section{3 | RESULTS AND DISCUSSION}

\section{1 | LC-MS/(MS) analysis}

\subsection{1 | Mass spectrometric conditions}

Instrumental parameters in MS and MS/MS were optimized by infusing the standard of SR9009 and of phase I metabolites available in our laboratory (M2 and M9) dissolved in methanol at a concentration of $10 \mathrm{\mu g} / \mathrm{mL}$. The full-scan MS analysis was first performed, both in positive and negative ionization mode, to identify the molecular ion. The best response was obtained in positive mode for the protonated molecular ion $[\mathrm{M}+\mathrm{H}]^{+}$at $\mathrm{m} / \mathrm{z} 438$ for SR9009 and at m/z 314 (M2) and at $\mathrm{m} / \mathrm{z} 283$ (M9) for the two metabolites available in our laboratory. The method was then optimized evaluating different mass spectrometric parameters.

For the triple quadrupole assays, optimal results were obtained using a curtain gas pressure of $25 \mathrm{psi}$, a source temperature of $500^{\circ} \mathrm{C}$, an ion source gas 1 pressure of $35 \mathrm{psi}$, an ion source gas 2 pressure of $40 \mathrm{psi}$, a declustering voltage of $60 \mathrm{~V}$, and a needle voltage of $5500 \mathrm{~V}$; while for the TOF assays the best conditions were obtained using a fragmentor voltage of $150 \mathrm{~V}$, an ion source temperature of $350^{\circ} \mathrm{C}$, a capillary voltage of $4000 \mathrm{~V}$, a nebulizer gas pressure of $45 \mathrm{psi}$ and a flow of the drying gas of $10 \mathrm{~L} / \mathrm{min}$.

Subsequently, MS/MS experiments were carried out at different collision energies $(20,30$, and $50 \mathrm{eV})$ to obtain information about the dissociation patterns of SR9009 and to select representative product ions to be used to set up the SRM acquisition method. The protonated molecular ion $[\mathrm{M}+\mathrm{H}]+$ undergoes extensive dissociation at a collision energy of $30 \mathrm{eV}$, as result of the tertiary amine cleavage, leading to the formation of an abundant product ion at m/z 125 ((4-chlorophenyl) methyl group) and of minor product ions at $\mathrm{m} / \mathrm{z}$ 142 ((5-nitrothiophen-2-yl) methyl moiety) and 154 ([(1ethoxycarbonyl)pyrrolidin-3-yl] methyl moiety) (Figure 1). At lower collision energy, a product ion at $\mathrm{m} / \mathrm{z} 392$ corresponding to the cleavage at level of the 1-pyrrolidinecarboxy acid moiety with the loss of the ethoxy group (Figure 1) was also detected.

The described product ions were utilized as diagnostic ions (structural markers: characteristic portions of the molecular structure that is common to SR9009 and its metabolites) to obtain structural information of the analytes detected in either the samples obtained after incubation of SR9009 in the presence of HLM or the samples collected after oral administration of a nutritional supplement containing SR9009.

\subsection{Characterization of the biotransformation pathways of SR9009}

The in vitro phase I metabolism protocol was set up by considering the data already reported in the literature ${ }^{27,28}$ and the protocols previously optimized in our laboratory to carry out similar in vitro studies. ${ }^{17-19,24}$ The best results were obtained using as substrate solvent DMSO (the total amount of DMSO in the final assay was $1 \%$ ), a substrate concentration of $1 \mathrm{mg} / \mathrm{mL}$, a protein concentration of $0.5 \mathrm{mg} /$ $\mathrm{mL}$, phosphate buffer $0.1 \mathrm{M}$ at $\mathrm{pH} 7.4$ and an incubation time of 4 hours.

The analysis of the in vitro samples using the TOF system in full scan as acquisition mode allowed to detect 10 compounds not found in the negative control sample obtained by incubating SR9009 in the absence of HLM, and two products (M2 and M4) detected also in the sample incubated in the absence of HLM, and therefore generated by both enzymatic and non-enzymatic reactions (Figure $2 \mathrm{~A}$ ). All the metabolites with the exception of metabolite M2 contain the characteristic chlorine isotope pattern (Table 1 shows the elemental composition). Samples were then analyzed by the triple quadrupole system in SRM as acquisition mode to obtain structural information of the analytes detected (Figure 2B). The ion transitions utilized to develop the SRM method (Table 1) were obtained by calculating the protonated molecular ion $[\mathrm{M}+\mathrm{H}]^{+}$of the potential SR9009 metabolite and by selecting the diagnostic product ions (structural markers) found in the product ion spectrum of the parent compound (Figure 1).

In detail, we have obtained the following results (Figure 2A,B shows the extracted ion chromatograms and Figure 3 the proposed chemical structures):

- Three chromatographic peaks (M1, M5, and M11) presenting precursor ion at $\mathrm{m} / \mathrm{z} 313$. The presence of product ions at $\mathrm{m} / \mathrm{z} 125$ and 170 and the absence of the product ions at m/z 142 and 154 , denote the loss of the (5-nitrothiophen-2-yl) methyl moiety and the hydroxylation of the ([(1-ethoxycarbonyl)pyrrolidin-3-yl] methyl portion.

- A chromatographic peak (M2) with precursor ion at $\mathrm{m} / \mathrm{z} 314$. The presence of representative product ions at $\mathrm{m} / \mathrm{z} 142$ and 154 and the absence of the characteristic chlorine isotope pattern denote the loss of the (4-chlorophenyl) methyl moiety.

- Three chromatographic peaks (M3, M4, and M10) with precursor ion at $\mathrm{m} / \mathrm{z} 297$. The presence of the characteristic chlorine isotope pattern and of the product ions at m/z 142 and 141 and the absence of the product ions at m/z 125 and 154 for $M 3$ and M10 denote the loss of the ([(1-ethoxycarbonyl)pyrrolidin-3-yl] methyl moiety and the oxidation of the (4-chlorophenyl) methyl moiety; whereas for M4 the absence of the product ion at $\mathrm{m} / \mathrm{z}$ 142 denote the loss of the (5-nitrothiophen-2-yl) methyl moiety;

- A chromatographic peak (M6) with precursor ion at $\mathrm{m} / \mathrm{z} 366$. The presence of productions at m/z 125 and 142 together with the absence of the product ion at $\mathrm{m} / \mathrm{z} 154$ denote a modification at level of the ([(1-ethoxycarbonyl)pyrrolidin-3-yl] methyl portion. The difference of 72 amu can be in fact explained with the loss of the ethoxycarbonyl group. 


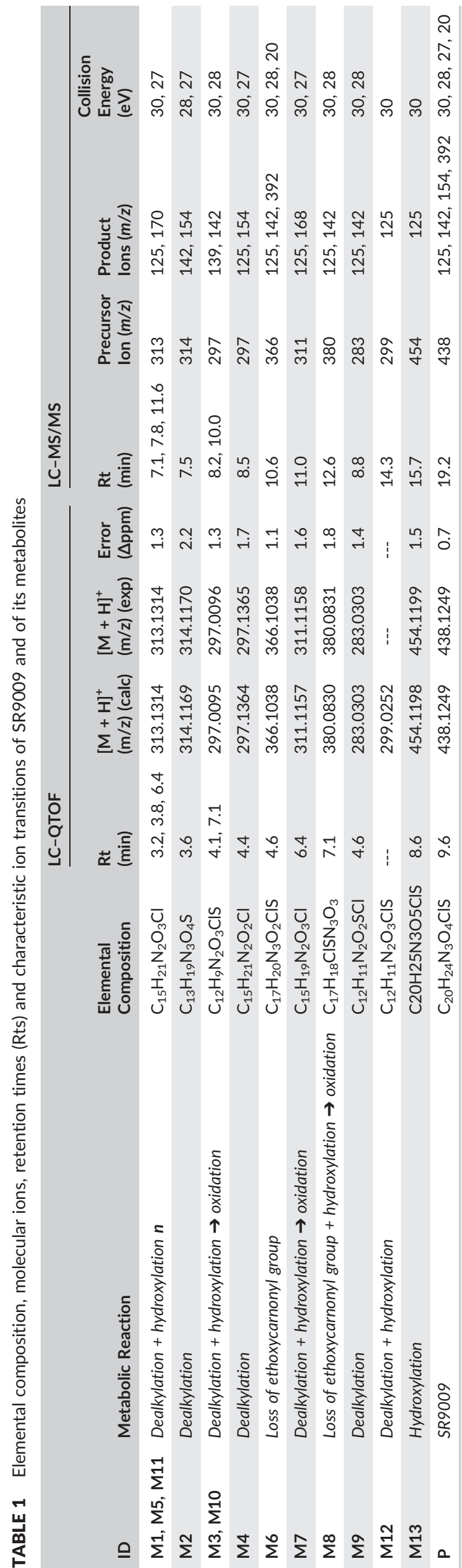

- A chromatographic peak (M7) with precursor ion at $\mathrm{m} / \mathrm{z}$ 311. The presence of product ions at $\mathrm{m} / \mathrm{z} 125$ and 170 together with the absence of the product ions at $\mathrm{m} / \mathrm{z} 142$ and 154 denote the loss of the (5-nitrothiophen-2-yl) methyl moiety and the oxidation of the ([(1-ethoxycarbonyl)pyrrolidin-3-yl] methyl portion.

- A chromatographic peak (M8) with precursor ion at $\mathrm{m} / \mathrm{z} 380$. The presence of product ion at $\mathrm{m} / \mathrm{z} 125$ and the absence of the product ions at m/z 142 and 154 denote modification at level of both the ([(1-ethoxycarbonyl)pyrrolidin-3-yl] methyl and the 2-methyl5-nitrothiophene portions. The loss of the ethoxycarbonyl group and the oxidation of ((5-nitrothiophen-2-yl) methyl portion can be supposed.

- A chromatographic peak (M9) with precursor ion at m/z 283; The presence of the product ions at $\mathrm{m} / \mathrm{z} 125$ and 142 and the absence of the product ion at $\mathrm{m} / \mathrm{z} 154$ denote the loss of the ([(1ethoxycarbonyl)pyrrolidin-3-yl] methyl group.

- A chromatographic peak (M12) with precursor ion at $\mathrm{m} / \mathrm{z} 299$. The presence of the characteristic chlorine isotope pattern and of the product ions at $\mathrm{m} / \mathrm{z} 142$ and 141 and the absence of the product ions at $\mathrm{m} / \mathrm{z} 125$ and 154 denote the loss of the 1pyrrolidinecarboxy acid moiety and the hydroxylation of the (4chlorophenyl) methyl moiety.

- A chromatographic peak (M13) with precursor ion at $\mathrm{m} / \mathrm{z} 454$; being the precursor ion at $\mathrm{m} / \mathrm{z} 454,16$ amu higher than the precursor ion of SR9009 (m/z 438), the detected peak was assigned as hydroxylated metabolite.

To define the relative contribution of individual CYPs isoforms to the overall metabolic rate of observed biotransformation, SR9009 (at a concentration of $1 \mathrm{mg} / \mathrm{mL}$ ) was incubated with the cDNA expressed CYP450 isoforms selected in this study. The CYP450 isoforms involved in the phase I metabolism of SR9009 are the CYP3A4, the CYP3A5, the CYP2C19 and the CYP2D6 (Table 2). To confirm these results, SR9009 was then incubated with pooled human liver microsomes in the presence of specific inhibitors, added to a final concentration of $1 \mathrm{mg} / \mathrm{mL}$, of CYP3A (ketoconazole), CYP2C19 (fluconazole), and CYP2D6 (paroxetine) isoforms. The results showed that in the presence of ketoconazole and paroxetine a significant decrease in the of phase I metabolites was registered, while moderate variations were measured in the presence of fluconazole, significantly influencing only the formation of M2, M4, and M9 (Table 2).

Concerning the phase II metabolism, most of the phase I metabolites identified in this study also underwent moderate (lower than $20 \%$ ) glucuronidation (data not shown).

\section{3 | Effects of gender, genetic polymorphism, and drug-drug interaction on the metabolic profile of SR9009}

\subsection{1 | Gender, genetic polymorphism}

To investigate the effect of gender and genetic polymorphism in altering the in vitro phase I metabolic profile of SR9009, in vitro assays were performed incubating SR9009 (a) in the presence of either 

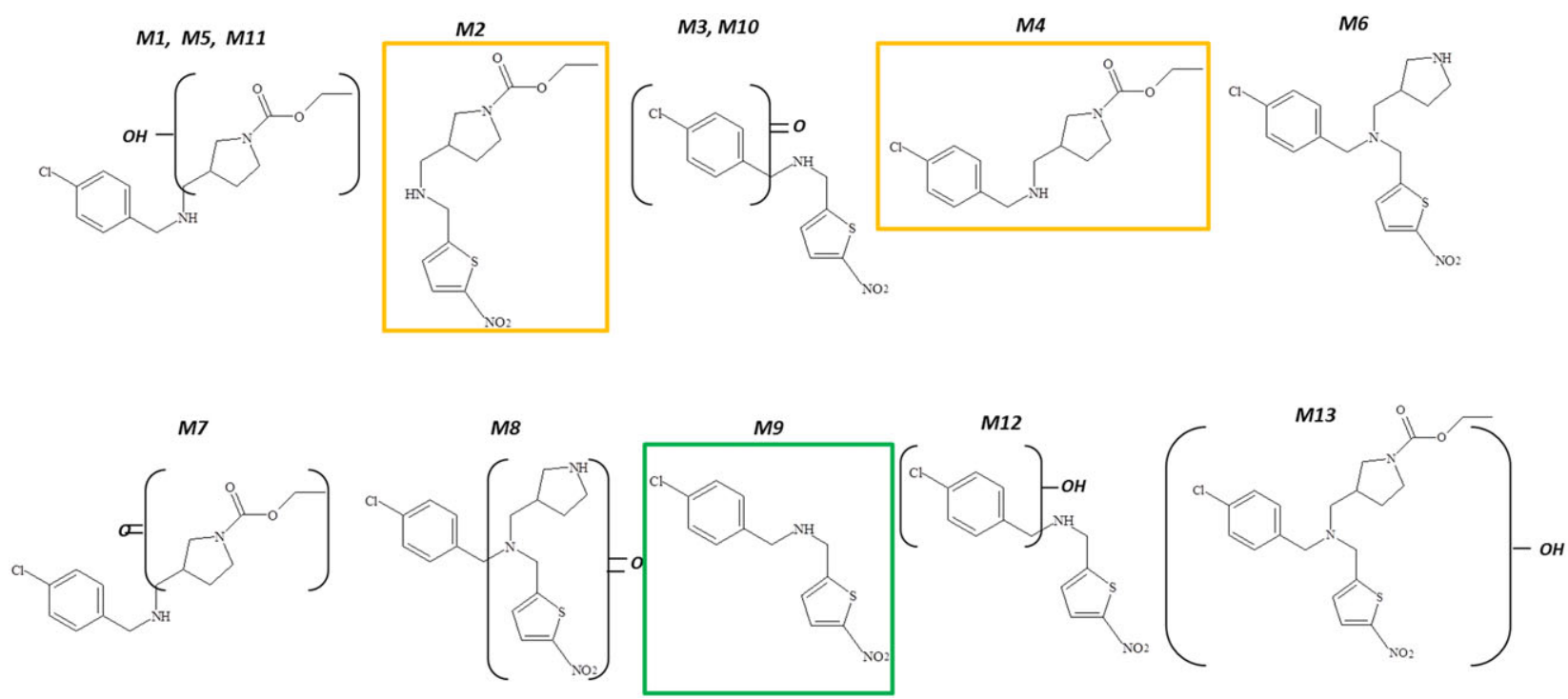

metabolites produced by both enzymatic and non-enzymatic reactions

- metabolite selected by the WADA as the most appropriate marker of SR9009 use

FIGURE 3 Proposed molecular structures of the SR9009 metabolites identified in this study

TABLE 2 Enzymatic isoforms involved in the phase I metabolism of SR9009

\begin{tabular}{|c|c|c|c|c|}
\hline ID & CYP $3 A 4$ & CYP 3 A5 & CYP 2 C19 & CYP 2D6 \\
\hline M1 & - & - & + & + \\
\hline M2 & + & + & + & - \\
\hline M3 & + & + & + & + \\
\hline M4 & + & + & + & - \\
\hline M5 & + & + & + & + \\
\hline M6 & + & + & + & + \\
\hline M7 & + & + & - & - \\
\hline M8 & - & - & + & + \\
\hline M9 & + & + & + & + \\
\hline M10 & + & + & - & + \\
\hline M11 & + & + & - & - \\
\hline M12 & + & + & - & + \\
\hline M13 & + & + & + & - \\
\hline
\end{tabular}

HLM from female or male donors to investigate gender differences, or (b) in the presence of HLM containing the different CYP2D6 or CYP2C19 allelic variants to evaluate the genetic differences. The results obtained incubating SR9009 in the presence of HLM from either female or male donors showed no appreciable differences (data not shown) between the respective metabolic profiles. Concerning the influence of genetic polymorphisms, Figure $4 \mathrm{~A}-\mathrm{C}$ reports the results obtained incubating SR9009 in the presence of HLM containing the different CYP2D6 allelic variants (high activity, moderate activity, and without activity). As can be noticed, a significant alteration of the metabolic profile of SR9009 was registered. In detail, only in the presence of the allelic variant with high activity, the metabolic profile was found to be complete; in the other two cases, only three metabolites were formed (M2, M4, and M9). Different results were instead obtained using the CYP2C19 allelic variants. The formation of the metabolites M2 and M4 was high only in the presence of the allelic variant with high activity, whereas the other metabolites were not influenced by the different activity of the CYP2C19 isoenzyme (data not shown).

\subsection{2 | Drug-drug interaction}

To investigate the potential effects of drug-drug interaction in altering the in vitro phase I metabolic profile of SR9009, inhibition studies were performed incubating SR9009 with either HLM or with the cDNA expressed CYP450 isoforms (CYP3A4, CYP3A5, CYP2C19, and CYP2D6) mainly involved in the biotransformation pathways observed, in the absence and in the presence of the non-prohibited drugs selected in this study (four antifungals: ketoconazole, miconazole, itraconazole, fluconazole; three antidepressants: paroxetine, fluoxetine, nefazodone; three non-steroidal anti-inflammatory drugs: ibuprofen, ketoprofen, diclofenac; two $\mathrm{H} 2$ receptor antagonist agents: ranitidine, cimetidine; two steroidal progestins: gestodene or levonorgestrel) using the same in vitro protocol and the analytical procedures already described in Section 2. Figure 5A-D shows the results obtained incubating SR9009 $(1 \mathrm{mg} / \mathrm{mL})$ with HLM in the absence (control, (A)) and in the presence of $1 \mathrm{mg} / \mathrm{mL}$ of ketoconazole (B) miconazole (C), itraconazole (D) or fluconazole (E). The levels of all the metabolites were significantly reduced (> $20 \%$ ) by miconazole and ketoconazole; moderate variation was registered in the presence of itraconazole, whereas in the presence of fluconazole only the production of the metabolites formed by the CYP2C19 was influenced. Concerning the other inhibitors tested, significant alteration in the metabolic profile of SR9009 was registered in the presence of 


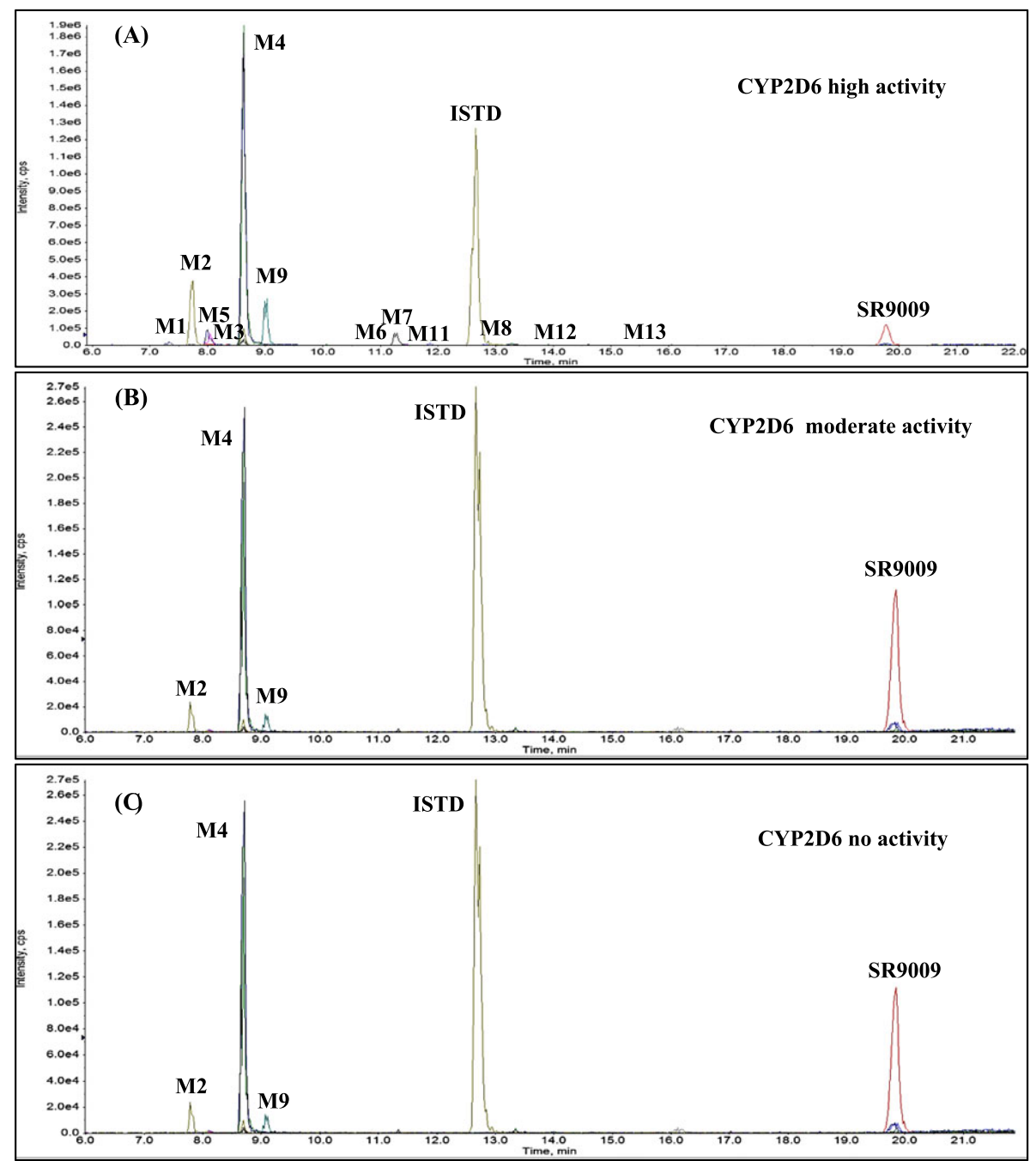

FIGURE 4 LC-MS/MS extracted ion chromatograms of the phase I in vitro samples obtained after incubation of SR9009 in the presence of HLM containing the different CYP2D6 allelic variants: high activity (A), moderate activity (B) and no activity (C)

nefazodone, paroxetine and fluoxetine (data not shown); whereas moderate modifications were measured in the presence of the steroidal progestins and no effects were registered in the presence of the $\mathrm{H} 2$ receptor antagonist agents and of the NSAIDs considered in this study (data not shown). To assess whether the observed inhibitory effects were dose dependent, SR9009 was incubated in the presence of different concentrations $(0.001,0.002,0.01,0.1,0.2,0.5,1$, and $10 \mathrm{mg} / \mathrm{mL}$ ) of the antifungals, steroidal progestins or antidepressants considered in this study, using either HLM or the cDNA expressed isoforms mainly involved in the metabolism of SR9009 (CYP3A4, CYP2C19, and CYP2D6). Figure 6 shows the curves obtained plotting the relative enzymatic activity (control $=100 \%$ ) of the CYP450 isoenzymes involved in the formation of M2, M4, and M9 (the most abundant metabolites detected in the human urine) versus the concentration of inhibitor. As can be noticed, the formation of M2 and M9 is reduced by more than $50 \%$ (IC50) in the presence of miconazole and ketoconazole at a concentration of 0.002 and in the presence of fluconazole at a concentration of $0.01 \mathrm{mg} / \mathrm{mL}$; whereas in the presence of itraconazole significant differences (> 20\%) were registered only for the metabolite M9. The effects of inhibition on M4 were instead more evident in the presence of ketoconazole and fluconazole being CYP2C19 the isoenzyme responsible for the formation of this metabolite. Indeed, M4 was reduced by more than $50 \%$ (IC50) at a concentration of $0.01 \mathrm{mg} / \mathrm{mL}$ in the presence of fluconazole and ketoconazole, at a concentration of $0.1 \mathrm{mg} / \mathrm{mL}$ in the presence of miconazole, and at concentration higher than $1 \mathrm{mg} / \mathrm{mL}$ in the presence of itraconazole.

Concerning the results obtained in the presence of the antidepressants, the formation of $\mathrm{M} 2, \mathrm{M} 4$, and $\mathrm{M} 9$ was reduced by more than $50 \%$ (IC50) in the presence of nefazodone at a concentration of $0.5 \mathrm{mg} / \mathrm{mL}$. Paroxetine and fluoxetine, being the CYP2D6 involved minimally in the formation of these metabolites, caused moderate alteration only at the highest concentration tested (data not shown). Finally, in the presence of the steroidal progestins the formation of M2, M4, and M9 was reduced by more than 50\% (IC50) at the highest concentration tested $(10 \mathrm{mg} / \mathrm{mL}$ ) (data not shown).

\subsection{Case study - Analysis of excretion urine}

A urine sample collected after oral administration of a nutritional supplement containing SR9009, kindly supplied by WAADS was analyzed 

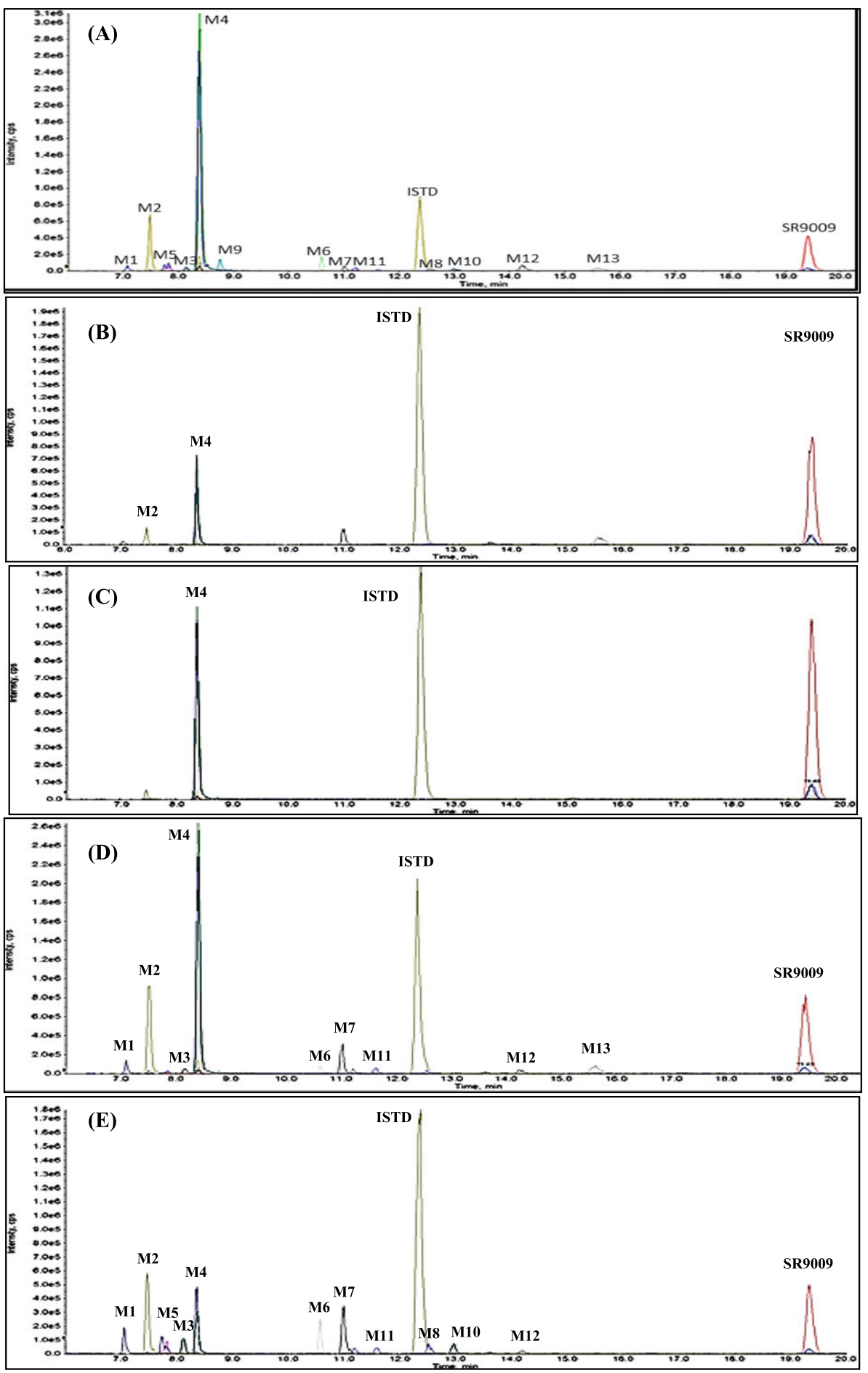

FIGURE 5 LC-MS/MS extracted ion chromatograms of the phase I in vitro samples obtained after incubation of SR9009 with human liver microsomes: in the absence (control, A), and in the presence of ketoconazole (B), miconazole (C), itraconazole (D) or fluconazole (E)

utilizing the analytical procedures developed in this study and used to analyze the in vitro samples. Figure $7 \mathrm{~A}-\mathrm{C}$ shows the extracted ion chromatograms of a negative urine (Figure $7 \mathrm{~A}$ ) and of the free (Figure 7B) and glucuronide (Figure 7C) fraction of the urine sample collected after administration of SR9009. Five metabolites (M2, M4, $\mathrm{M} 5, \mathrm{M} 8$, and $\mathrm{M9}$ ) were identified in the free fraction and eight (M1, M2, M4, M5, M7, M8, M9, and M12) in the glucuronide fraction. The percentage of glucuronidation was higher than $50 \%$ for all the metabolites detected, in contrast to the data obtained in vitro, where the percentage of glucuronidation was lower than $20 \%$.

The loss of the ([(1-ethoxycarbonyl)pyrrolidin-3-yl] methyl group (M9) followed by the loss of (5-nitrothiophen-2-yl) methyl group (M4), or the (4-chlrophenyl) methyl (M2) portion resulted to be the most important biotransformation pathways of SR9009 in human, confirming in part the results obtained in vitro, where M2 and M4 resulted to be the most abundant metabolites. 

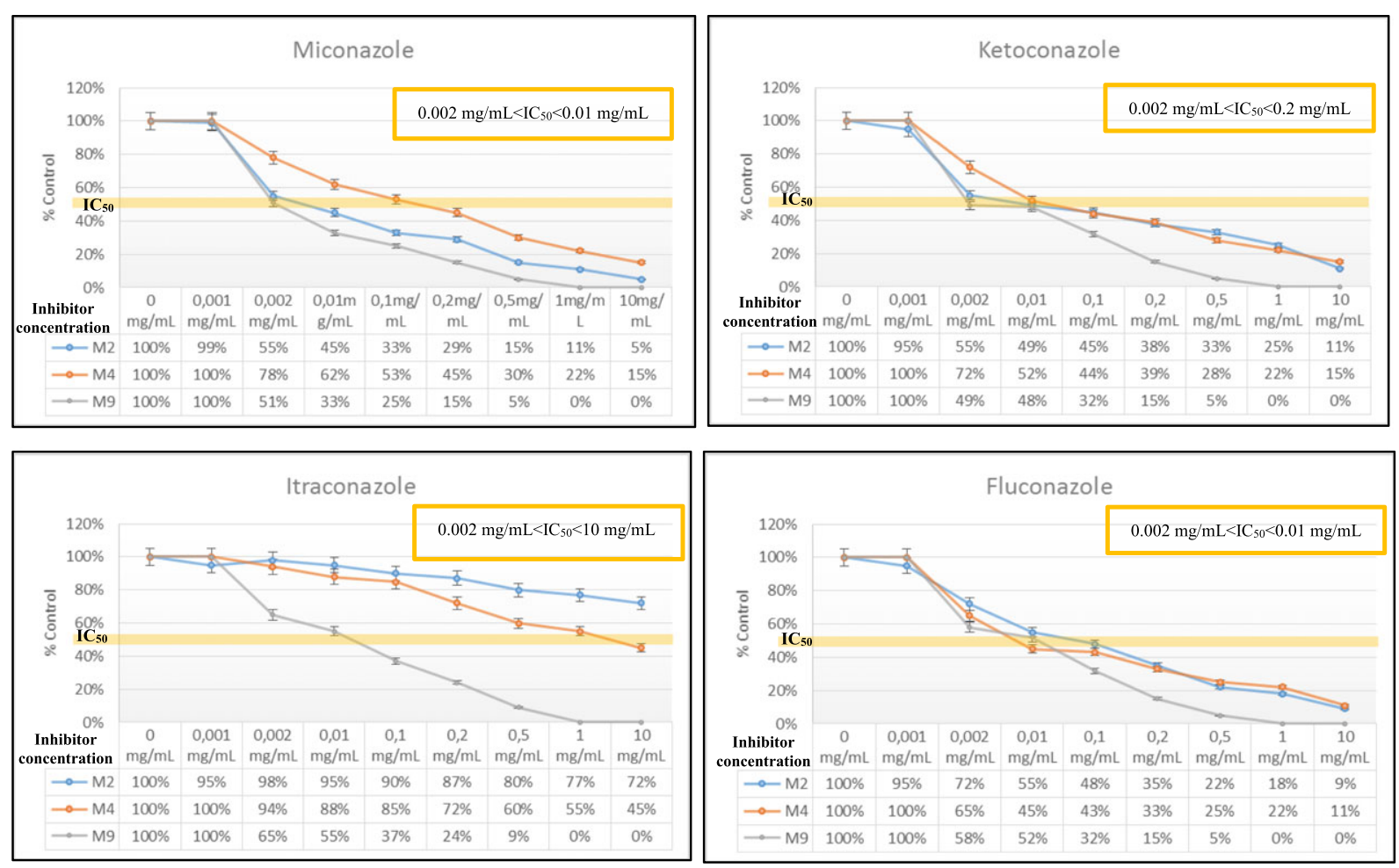

FIGURE 6 Alteration of the formation of M2, M4, and M9, in the absence and in the presence of different concentrations (0.001, 0.002, 0.01, $0.1,0.2,0.5,1$, and $10 \mathrm{mg} / \mathrm{mL}$ ) of the antifungals considered, utilizing the in vitro assays and the analytical procedure (pre-treatment and instrumental conditions) developed in this study. Each point represents the mean value of three independent determinations \pm the standard deviation of the analytical method

(A)

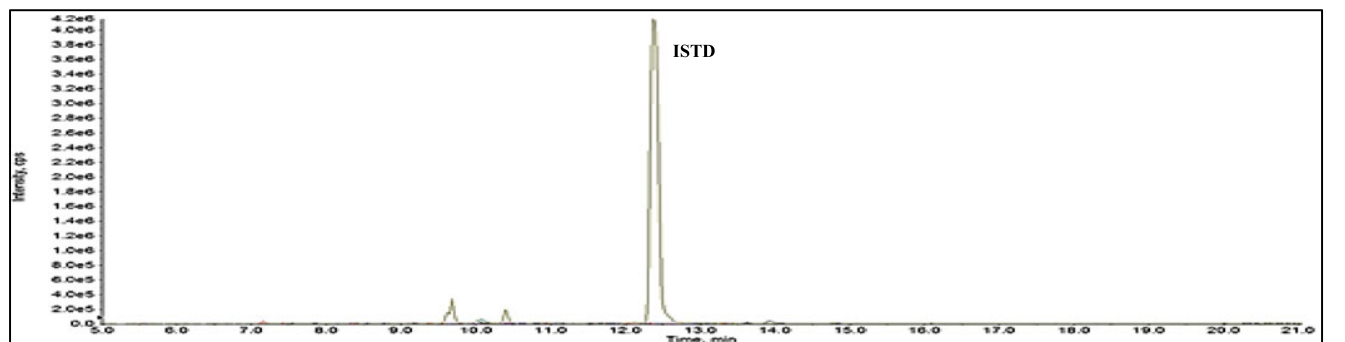

(B)

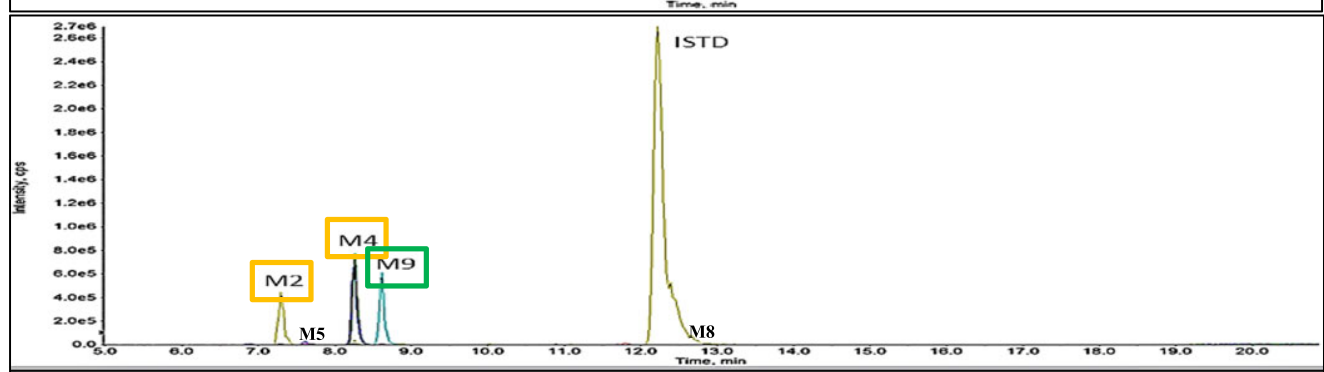

(C)

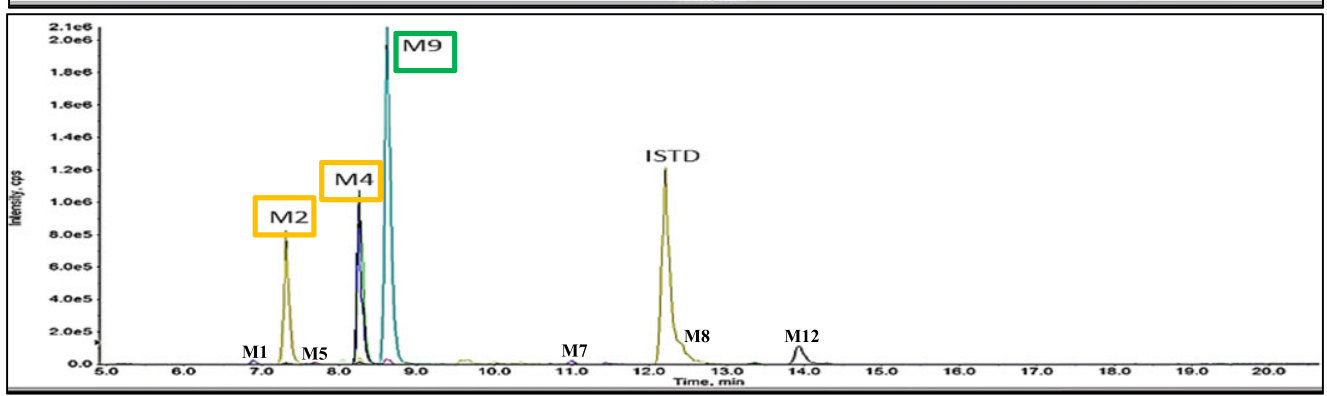

FIGURE 7 Extracted ion chromatograms obtained analyzing a negative urine (A) and a urine sample collected after oral administration of a nutritional supplement containing SR9009: free (B) and glucuronide (C) fraction 


\section{4 | CONCLUSIONS}

The analytical strategy and the in vitro protocols here described allowed to identify 13 metabolites for SR9009, several of which (M3, M10, and M12), to the best of our knowledge, have never been reported before. The phase I metabolic reactions include hydroxylation, oxidation de-alkylation, and a combination thereof, the dealkylated metabolites being the most abundant detected both in vitro and in the urine of the controlled excretion study. The enzymatic isoforms mainly involved in the SR9009 phase I biotransformation pathways are CYP3A4, CYP3A5, CYP2C19, and CYP2D6. Once formed, most of the phase I metabolites underwent phase II reactions (glucuronidation). No significant differences in the phase I metabolic profile were registered after incubation of SR9009 in the presence of either pooled female or male HLM, while significant differences were registered in the presence of different allelic variants of the enzymatic isoforms CYP2D6 and CYP2C19.

Concerning the effects of drug-drug interaction, the metabolic profile of SR9009 was significantly altered in the presence of ketoconazole, miconazole, fluoxetine, paroxetine, and nefazodone. Moderate differences were instead registered in the presence of fluconazole, itraconazole, gestodene, and levonorgestrel.

The evidence obtained in this study confirms the need of taking into due account both genetic polymorphism and drug-drug interaction to select the most appropriate marker of use in doping control analysis. WADA has already indicated the metabolite M9as the most appropriate marker of intake of SR9009. The results of the present study suggest that the metabolites M2 and M4 must also be considered, being generated by both enzymatic and non-enzymatic reactions.

\section{ACKNOWLEDGEMENT}

This project has been supported by a research grant by the Italian Department of Health (Ministero della Salute, Commissione per la vigilanza sul doping e sulla tutela sanitaria delle attività sportive).

\section{ORCID}

Monica Mazzarino (D) http://orcid.org/0000-0003-2036-930X

Xavier de la Torre (D) http://orcid.org/0000-0001-8037-6750

Francesco Botrè (i) http://orcid.org/0000-0001-5296-8126

\section{REFERENCES}

1. Everett LJ, Lazar MA. Nuclear Receptor Rev-erba: Up, Down, and All Around. Trends Endocrinol Metab. 2014;25(11):586-592.

2. Fang B, Lazar MA. Dissecting the Rev-erba cistrome and mechanisms controlling circadian transcription in liver. Cold Spring Harb Symp Quant Biol. 2015;80:233-238.

3. Yin L, Wu N, Lazar MA. Nuclear receptor Rev-erba: a heme receptor that coordinates circadian rhythm and metabolism. Nucl Recept Signal. 2010;8:e001.

4. Marciano DP, Chang MR, Corzo CA, et al. The Therapeutic Potential of Nuclear Receptor Modulators for Treatment of Metabolic Disorders: PPARs, RORs, REV-ERBs. Cell Metab. 2014;19(2):193-208.

5. Solt LA, Wang Y, Banerjee S, et al. Regulation of circadian behavior and metabolism by synthetic REV-ERB agonists. Nature. 2012;485:62-69.
6. Matta-Camacho E, Banerjee S, Hughes TS, et al. Structure of REVERB $\beta$ ligand-binding domain bound to a porphyrin antagonist. J Biol Chem. 2014;289:20054-20066.

7. Kojetin DJ, Burris TP. REV-ERB and ROR nuclear receptors as drug targets. Nat Rev Drug Discov. 2014;13(3):197-216.

8. Amador A, Huitron-Resendiz S, Roberts AJ, Kamenecka TM, Solt LA, Burris TP. Pharmacological Targeting the REV-ERBs in Sleep/Wake Regulation. PloS One. 2016;11(9):e0162452.

9. Amador A, Kamenecka TM, Solt LA, Burris TP. REV-ERB $\beta$ is required to maintain normal wakefulness and the wake-inducing effect of dual REV-ERB agonist SR9009. Biochem Pharmacol. 2018;150:1-8.

10. Fan W, Atkins AR, Yu RT, Downes M, Evans RM. Road to exercise mimetics: targeting nuclear receptors in skeletal muscle. J Mol Endocrinol. 2013;51(3):T87-T100.

11. Woldt E, Sebti Y, Solt LA, et al. Rev-erb-alpha modulates skeletal muscle oxidative capacity by regulating mitochondrial biogenesis and autophagy. Nat Med. 2013;19(8):1039-1046.

12. Thevis $M$, Schänzer W. Emerging drugs affecting skeletal muscle function and mitochondrial biogenesis-Potential implications for sports drug testing programs. Rapid Communications in Mass Spectrometry. 2016;30:635-651.

13. Miller R. Stenabolic (SR9009) Review-Game Changing Supplement? At https://www.supplementcritique.com/stenabolic-sr9009-reviewgame-changing-supplement/

14. World Anti-Doping Agency. The 2018 Prohibited List. International Standard, Montreal, 2018. Available at: www.wada-ama.org. (last accessed: March 5th 2018).

15. Sobolevsky T, Dikunets M, Rodchenkov G. Metabolism study of GSK4112, SR9009, and SR9011, new "exercise-in-a-pill" drug candidates. In: Schänzer W, Thevis M, Geyer H, Mareck U, eds. Recent Advances in Doping Analysis (23). Cologne, Germany: Sport \& Buch Strauß; 2015:64-69.

16. Geldof L, Deventer K, Roels K, Tudela E, Van Eenoo P. In vitro metabolic studies of REV-ERB agonists SR9009 and SR9011. Int J Mol Sci. 2016;17(10):1676-1688.

17. Mazzarino M, de la Torre X, Fiacco I, Palermo A, Botrè F. Drug-drug interaction and doping, part 1: A In Vitro study on the effect of nonprohibited Drugs on the phase I metabolic profile of toremifene. Drug Test Anal. 2014;6:482-491.

18. Mazzarino $M$, de la Torre $X$, Fiacco I, Botrè F. Drug-drug interaction and doping, part 2: An in vitro study on the effect of non-prohibited drugs on the phase I metabolic profile of stanozolol. Drug Test Anal. 2014;6:969-977.

19. Mazzarino M, Buccilli V, de la Torre $X$, et al. Characterization of the phase I and phase II metabolic profile of tolvaptan by in vitro studies and liquid chromatography-mass spectrometry profiling: relevance to doping control analysis. J Pharm Biomed Anal. 2017;145:555-568.

20. Niwa T, Shiraga T, Takagi A. Effect of antifungal drugs on cytochrome P450 (CYP) 2C9, CYP2C19, and CYP3A4 activities in human liver microsomes. Biol Pharm Bull. 2005;28(9):1805-1808.

21. Greenblatt DJ, Zhao Y, Venkatakrishman K, et al. Mechanism of cytochrome P450-3A inhibition by ketoconazole. J Pharm Pharmacol. 2011;63:214-221.

22. Ouellet D, Bramson C, Roman D, et al. Effects of three cytochrome P450 inhibitors, ketoconazole, fluconazole, and paroxetine, on the pharmacokinetics of lasofoxifene. Br J Clin Pharmacol. 2007;63(1): 59-66.

23. Ereshefsky L, Riesenman C, Lam YM. Antidepressant drug interactions and the cytochrome P450 system. The role of cytochrome P450 2D6, Clin Pharmacokinet 1995;29:10-18.

24. Mazzarino $M$, Biava $M$, de la Torre $X$, Fiacco I, Botrè F. Characterization of the biotransformation pathways of clomiphene, tamoxifen and toremifene as assessed by LC-MS/(MS) following in vitro and excretion studies. Anal Bioanal Chem. 2013;405(16):5467-5487. 
25. Mazzarino $M$, de la Torre $X$, Botrè $F$. A screening method for the simultaneous detection of glucocorticoids, diuretics, stimulants, antioestrogens, beta-adrenergic drugs and anabolic steroids in human urine by LC-ESI-MS/MS. Anal Bioanal Chem. 2008;392(4):681-698.

26. Mazzarino M, Fiacco I, de la Torre $X$, Botrè $F$. A mass spectrometric approach for the study of the metabolism of clomiphene, tamoxifen and toremifene by liquid chromatography time-of-flight spectroscopy. Eur J Mass Spectrom. 2008;14(3):171-180.

27. Hill JR. In Vitro Drug Metabolism Using Liver Microsomes. In: Current Protocols in Pharmacology, Supp. 23, Section 7.8. John Wiley \& Sons, Inc; 2003:7.8.1-7.8.11.
28. Kuuranne T, Leinonen A, Schanzer W, Kamber M, Kostiainen R, Thevis M. Aryl Propionamide-Derived Selective Androgen Receptor Modulators: Liquid Chromatography-Tandem Mass Spectrometry Characterization of the in Vitro Synthesized Metabolites for Doping Control Purposes. Drug Metab Dispos. 2008;36(3):571-581.

How to cite this article: Mazzarino M, Rizzato N, Stacchini C, de la Torre $\mathrm{X}$, Botrè $\mathrm{F}$. A further insight into the metabolic profile of the nuclear receptor Rev-erb agonist, SR9009. Drug Test Anal. 2018;10:1670-1681. https://doi.org/10.1002/dta.2538 